\title{
Some Inferences on Semicircular Distribution
}

\author{
M. Ahsanullah \\ Rider University, 2083 Lawrenceville Road, Lawrenceville, \\ New Jersey, NJ 08648, USA \\ ahsan@rider.edu
}

Received 10 November 2015

Accepted 14 April 2016

\begin{abstract}
Several distributional properties of semicircular distribution are presented. The limiting distributions of the standardized extreme order statistics are given. Some characterizations of the distribution are shown.
\end{abstract}

\section{Introduction}

Wishart (1928) considered random matrix in connection to the statistical analysis of large data. A matrix is called a random matrix if the entries of the matrix are random numbers from a specified distribution. If the distribution is Gaussian, then we call it a Gaussian random matrix. Let $\lambda_{i}, i=1,2, \ldots, n$ be the eigenvalues of the matrix. The empirical spectral density (ESD) $m(\lambda)$ is defined as

$$
m(\lambda)=\frac{1}{n} \sum_{i=1}^{n} \delta\left(\lambda-\lambda_{i}\right)
$$

where $\delta$ is the Dirac delta function. The ESD is a probability measure over the complex plane. If the matrix is Hermitian, the eigenvalues are real and $m(\lambda)$ is a real probability density function. Wagner (1955) proved that the ESD of a Hermitian $n \times n$ Gaussian matrix when normalized by $\frac{1}{\sqrt{n}}$ tends to the semicircular distribution with the following $\operatorname{pdf} f_{1}(x)$ where

$$
f_{1}(x)=\frac{1}{2 \pi} \sqrt{4-x^{2}},-2<x<2 .
$$

This work has been extended by various authors. For details see Anderson et al. (2010), Bai and Yin (1988) and Tao (2012). The $\operatorname{pdf} f_{R}(x)$ of the generalized semicircular distribution is given by

$$
\frac{2}{\pi R^{2}} \sqrt{R^{2}-x^{2}},-R \leq x \leq R
$$

The pdf of the standardized $\operatorname{SCD}(-1,1) f_{\mathrm{s}}(x)$ is given by

$$
f_{\mathrm{s}}(x)=\frac{2}{\pi} \sqrt{1-x^{2}},-1 \leq x \leq 1 .
$$


Suppose $Y$ has a beta distribution with pdf $f(x)$ as

$$
f_{\mathrm{B}}(y)=\sqrt{y(1-y)}, 0<y<1,
$$

then $W=2 y-1$ is distributed as $\operatorname{SCD}(-1,1)$. The standard semicircular distribution is symmetric around zero. The mean of $\operatorname{SCD}(-1,1)$ is zero and variance $=1 / 4$.

\section{Main Results}

The cumulative distribution function $(\mathrm{CDF}) F_{\mathrm{s}}(x)$ of $\operatorname{SCD}(-1,1)$ is given by

$$
F_{\mathrm{s}}(x)=\frac{1}{2 \pi}\left(\pi+2 \arcsin x+2 x \sqrt{1-x^{2}}\right) .
$$

Figure 2.1 gives the pdf of $\operatorname{SCD}(-1,1)$-Black, $\operatorname{SCD}(-3,3)$-Red, $\operatorname{SCD}(-6,6)$-Green.

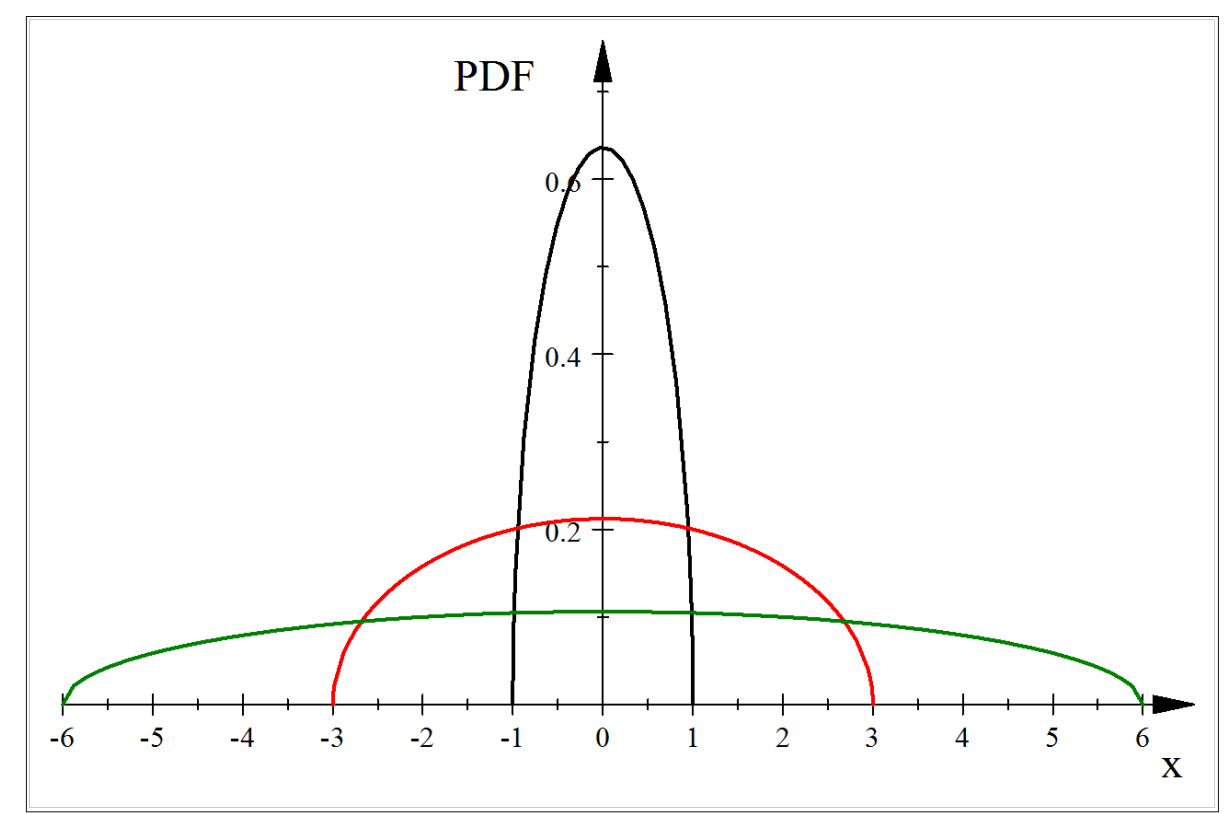

Figure 2.1.

The percentile points $x_{p}$ of $\operatorname{SCD}(-1,1)$ are given in Table 2.1 .

Table 2.1. Percentile points of $\operatorname{SCD}(-1,1)$.

\begin{tabular}{ll}
\hline$p$ & $x_{p}$ \\
\hline 0.1 & -0.68705 \\
0.2 & -0.49186 \\
0.3 & -0.31969 \\
0.4 & -0.15774 \\
0.5 & 0.0 \\
0.6 & 0.15774 \\
0.7 & 0.31969 \\
0.8 & 0.49186 \\
0.9 & 0.68705 \\
\hline
\end{tabular}


The hazard rate $h(z)$ of $\operatorname{SCD}(-1,1)$ is

$$
h(z)=\frac{4 \sqrt{1-x^{2}}}{\pi-2 \arcsin x-2 x \sqrt{1-x^{2}}}
$$

The hazard rate is monotone increasing. The moment generating function $M(t)$ is

$$
M(t)=\int_{-1}^{1} e^{t x} \frac{1}{2 \pi} \sqrt{1-x^{2}} d x=\frac{2 I_{1}(t)}{t}
$$

where $I_{1}(t)$ is the modified Bessel function. Let $X_{1}, X_{2}, \ldots, X_{n}$ be an independent observation from $\operatorname{SCD}(-1$, 1). Suppose $X_{1, n} \leq X_{2, n} \leq \ldots \leq X_{n, n}$ is the associated order statistics. It can be shown that

$$
\lim _{n \rightarrow \infty} \frac{F^{-1}\left(\frac{1}{n}\right)-F^{-1}\left(\frac{2}{n}\right)}{F^{-1}\left(\frac{2}{n}\right)-F^{-1}\left(\frac{4}{n}\right)}=2^{-1 . / 2}
$$

Thus $X_{1, n}$ when normalized tends to the extreme Value type III (minimum ) distribution (for details see Ahsanullah and Nevzorov (2001). Further

$$
\lim _{n \rightarrow \infty} P\left(X_{1, n} \leq a_{n}+b_{n} x\right)=1-e^{-\sqrt{x}}, x \geq 0, a_{n}=-1 \text { and } b_{n}=n^{-2 / 3}
$$

It can also be shown that

$$
\lim _{n \rightarrow \infty} \frac{F^{-1}\left(1-\frac{1}{n}\right)-F^{-1}\left(1-\frac{2}{n}\right)}{F^{-1}\left(1-\frac{2}{n}\right)-F^{-1}\left(1-\frac{4}{n}\right)}=2^{-1 . / 2}
$$

Thus $X_{n, n}$ when normalized tends to the extreme Value type III (maximum) distribution. We have

$$
\lim _{n \rightarrow \infty} P\left(X_{n, n} \leq a_{n}+b_{n} x\right)=e^{-\sqrt{(-x)}}, x \leq 0, a_{n}=-1 \text { and } b_{n}=n^{-2 / 3} .
$$

The following two lemmas will be used to prove the characterizing theorems.

Lemma 2.1. Suppose the random variable $X$ has an absolutely continuous $c d f F(x)$ and $p d f f(x)$. We assume $\alpha$ $=\inf \{X \mid F(x)>0\}, \beta=\sup \{x \mid F(x)<1\}, E(X)$ exists and $f(x)$ is differentiable for all $x$ in $(\alpha, \beta)$. If $E(X \mid X \leq x)$ $=g(x) \tau(x), g(x)$ is a differentiable function for all $x$ in $(\alpha, \beta)$ and $\tau(x)=\frac{f(x)}{F(x)}$, then

$$
f(x)=c e^{\int \frac{x-g^{\prime}(x)}{g(x)} d x},
$$

where $c$ is determined by the condition

$$
\int_{\alpha}^{\beta} f(x) d x=1
$$

Proof. We have

$$
E(X \mid X \leq x)=g(x) \tau(x)
$$

and 


$$
g(x)=\frac{\int_{\alpha}^{x} u f(u) d u}{f(x)}
$$

Thus

$$
\int_{\alpha}^{x} u f(u) d u=g(x) f(x) .
$$

Differentiating the above expression with respect to $x$, we obtain

$$
x f(x)=f^{\prime}(x) g(x)+f(x) g^{\prime}(x) .
$$

Thus

$$
\frac{f^{\prime}(x)}{f(x)}=\frac{x-g^{\prime}(x)}{g(x)} .
$$

On integrating the above expression, we will have

$$
f(x)=c e^{\int \frac{x-g^{\prime}(x)}{g(x)} d x},
$$

where $c$ is determined by the condition

$$
\int_{\alpha}^{\beta} f(x) d x=1 .
$$

Lemma 2.2. Suppose the random variable $X$ has an absolutely continuous $c d f F(x)$ and $p d f f(x)$. We assume $\alpha=\inf \{X \mid F(x)>0\}, \beta=\sup \{x \mid F(x)<1\}, E(X)$ exists and $f(x)$ is differentiable for all $x$ in $(\alpha, \beta)$. If $E(X \mid X \geq x)$ $=h(x) r(x), h(x)$ is a differentiable function for all $x$ in $(\alpha, \beta)$ and $r(x)=\frac{f(x)}{1-F(x)}$, then

$$
f(x)=c e^{-\int \frac{x+h^{\prime}(x)}{h(x)} d x}
$$

where $c$ is determined by the condition

$$
\int_{\alpha}^{\beta} f(x) d x=1
$$

Proof. We have

$$
E(X \mid X \geq x)=h(x) r(x)
$$

and

$$
h(x)=\frac{\int_{x}^{\beta} u f(u) d u}{f(x)}
$$

Thus

$$
\int_{x}^{\beta} u f(u) d u=h(x) f(x) .
$$

Differentiating the above expression with respect to $x$, we obtain

$$
x f(x)=f^{\prime}(x) h(x)+f(x) h^{\prime}(x) .
$$


Thus

$$
\frac{f^{\prime}(x)}{f(x)}=-\frac{x+h^{\prime}(x)}{h(x)}
$$

On integrating the above expression, we will have

$$
f(x)=c e^{-\int \frac{x-h^{\prime}(x)}{h(x)} d x},
$$

where $c$ is determined by the condition

$$
\int_{\alpha}^{\beta} f(x) d x=1 .
$$

Theorem 2.1. Suppose the random variable $X$ has an absolutely continuous $c d f F(x)$ and $p d f f(x)$. We assume $\alpha=-1$ and $\beta=1, f(x)$ is differentiable for all $x$ in $(-1,1)$ and $E(X)$ exists. Then

$$
E(X \mid X \leq x)=g(x) \frac{f(x)}{F(x)},
$$

where

$$
g(x)=\frac{x^{2}-1}{3} \text { if and only if } f(x)=\frac{2}{\pi} \sqrt{1-x^{2}},-1 \leq x \leq 1 .
$$

Proof. If

$$
f(x)=\frac{2}{\pi} \sqrt{1-x^{2}},
$$

then

$$
g(x)=\frac{\int_{-1}^{x} u \sqrt{1-u^{2}}}{\sqrt{1-x^{2}}}=\frac{x^{2}-1}{3} .
$$

Suppose

$$
g(x)=\frac{\int_{-1}^{x} u \sqrt{1-u^{2}}}{\sqrt{1-x^{2}}}=\frac{x^{2}-1}{3},
$$

then

$$
g^{\prime}(x)=2 x / 3 \text { and } \frac{x-g^{\prime}(x)}{g(x)}=\frac{-x}{1-x^{2}} .
$$

We have by Lemma 2.1,

$$
\frac{f^{\prime}(x)}{f(x)}=-\frac{x}{1-x^{2}} .
$$

On integrating both sides of the above equation, we obtain

$$
F(x)=c \sqrt{1-x^{2}},
$$


where $c$ is a constant. Using the boundary condition $\int_{-1}^{1} f(x) d x=1$, we obtain

$$
f(x)=\frac{2}{\pi} \sqrt{1-x^{2}},-1 \leq x \leq 1 .
$$

Theorem 2.2. Suppose the random variable $X$ has an absolutely continuous $c d f F(x)$ and $p d f f(x)$. We assume, $\alpha=-1$ and $\beta=1, f(x)$ is differentiable for all $x$ in $(-1,1)$ and $E(X)$ exists. Then

$$
E(X \mid X>x)=h(x) \frac{f(x)}{F(x)},
$$

where

$$
h(x)=\frac{x^{2}-1}{3} \text { if and only if } f(x)=\frac{2}{\pi} \sqrt{1-x^{2}},-1 \leq x \leq 1 .
$$

Proof. If

$$
f(x)=\frac{2}{\pi} \sqrt{1-x^{2}},
$$

then

$$
h(x)=\frac{\int_{x}^{1} u \sqrt{1-u^{2}}}{\sqrt{1-x^{2}}}=\frac{1-x^{2}}{3} .
$$

Suppose

$$
h(x)=\frac{1-x^{2}}{3}
$$

then

$$
h^{\prime}(x)=-2 x / 3 \text { and }-\frac{x+h^{\prime}(x)}{h(x)}=\frac{-x}{1-x^{2}} .
$$

We have by Lemma 2.2,

$$
\frac{f^{\prime}(x)}{f(x)}=-\frac{x}{1-x^{2}}
$$

On integrating both sides of the above equation, we obtain

$$
F(x)=c \sqrt{1-x^{2}}
$$

where $c$ is a constant. Using the boundary condition $\int_{-1}^{1} f(x) d x=1$, we obtain

$$
f(x)=\frac{2}{\pi} \sqrt{1-x^{2}},-1 \leq x \leq 1 .
$$


We define

$$
S_{k, n}=\frac{1}{k}\left(X_{1, n}+X_{2, n}+\cdots+X_{k, n}\right)
$$

and

$$
T_{k, n}=\frac{1}{k-1}\left(X_{k+1, n}+X_{k+2, n}+\cdots+X_{n, n}\right) .
$$

The following two characterization theorems are based on the conditional expectation of $S_{k, n}$ and $T_{k, n}$.

Theorem 2.3. Suppose the random variable $X$ has an absolutely continuous $c d f F(x)$ and $p d f f(x)$. We assume $\alpha=-1$ and $\beta=1, f(x)$ is differentiable for all $x$ in $(-1,1)$ and $E(X)$ exists. Then

$$
E\left(S_{k, n} \mid X_{k, n} \leq x\right)=g(x) \frac{f(x)}{F(x)},
$$

where

$$
g(x)=\frac{1-x^{2}}{3} \text { if and only if } f(x)=\frac{2}{\pi} \sqrt{1-x^{2}},-1 \leq x \leq 1 \text {. }
$$

Proof. We have

$$
E\left(S_{k, n} \mid X_{k, n}=x\right)=E(X \mid X \leq x),
$$

thus the proof follows from Theorem 2.1.

Theorem 2.4. Suppose the random variable $X$ has an absolutely continuous $c d f F(x)$ and $p d f f(x)$. We assume $\alpha=-1$ and $\beta=1, f(x)$ is differentiable for all $x$ in $(-1,1)$ and $E(X)$ exists. Then

where

$$
E\left(T_{k, n} \mid X_{k, n}=x\right)=h(x) \frac{f(x)}{F(x)},
$$

$$
h(x)=\frac{1-x^{2}}{3} \text { if and only if } f(x)=\frac{2}{\pi} \sqrt{1-x^{2}},-1 \leq x \leq 1 .
$$

Proof. We have

$$
E\left(T_{k, n} \mid X_{k, n}=x\right)=E(X \mid X \geq x),
$$

thus the proof follows from Theorem 2.2.

\section{References}

[1] M. Ahsanullah, V.B. Nevzorov, and M. Shakil, An Introduction to Order Statistics (Atlantis-Press, Paris, France, 2015)

[2] M. Ahsanullah and V.B. Nevzorov, Ordered Random Variables (Nova Science Publishers Inc., New York, USA, 2001).

[3] T.W. Anderson, A. Guiomet and O. Zeitoumi, An Introduction to Random Matrix (Cambridge University Press, Cambridge, U.K., 2010).

[4] Z.D. Bai and V. Yin, Converegence to Semi Circular Law, in Annals of Probability 16 (1988) 1729-1741.

[5] T. Tao, Topics in Random Matrix Theory (American Mathematical Society, Providence, R.I., USA, 2012).

[6] E.P. Wagner, Characteristic vectors of borthered matrices with infinite dimensions, Annals of Mathematics 52(2) (1955) 545564.

[7] J. Wishart, The generalized product moment distribution in samples from a normal distribution, Biometrika 28A (1928) 32-52. 